\title{
Caractérisation du système de production du gingembre (Zingiber officinale Rosc.) au Burkina Faso : Potentialités, contraintes et perspectives
}

\author{
Hervé NANDKANGRE $^{1 *}$, Mahama OUEDRAOGO ${ }^{1}$ et Mahamadou SAWADOGO ${ }^{2}$ \\ ${ }^{I}$ Institut de l'Environnement de Recherches Agricoles (INERA), \\ Département Productions Végétales, 04 BP 8645 Ouagadougou, Burkina Faso. \\ ${ }^{2}$ Université de Ouagadougou, UFR-SVT, Département de Biologie et Ecologie Végétales, \\ Laboratoire de Génétique et de Biotechnologie Végétales, 03 BP 7021 Ouagadougou 03, Burkina Faso. \\ *Auteur correspondant ; E-mail : hervenankangre@yahoo.fr; Tel: 002267204 30 62, Fax: 0022650340271
}

\section{RESUME}

Le gingembre est une herbacée annuelle. C'est l'une des plus importantes épices à travers le monde et est d'une importance économique avec de nombreuses vertus médicinales. Cette étude vise à caractériser le système de production du gingembre (Zingiber officinale Rosc.) au Burkina Faso dans le but de mieux faire connaître l'espèce et de mettre en exergue ses potentialités.. Les informations collectées à l'aide des fiches d'enquêtes ont permis de décrire avec exactitude la zone de production de l'espèce et de caractériser le système de production au Burkina Faso. Le gingembre a été introduit au Burkina Faso il y a plusieurs dizaines d'années et est communément appelé «gnamankou ». La plante est cultivée essentiellement dans la partie Sud-Ouest du pays où les précipitations annuelles sont comprises entre 900 et $1200 \mathrm{~mm}$. La culture du gingembre est peu répandue et se pratique généralement aux abords des bas-fonds sur de petites superficies. Les pratiques culturales adoptées ne suivent aucun itinéraire technique établit avec des moyens de production souvent dérisoires. Sa production est limitée malgré ses nombreuses potentialités médicinales et économiques. Les institutions en charge de l'agriculture ne disposent pas de données concernant la culture du gingembre.

(C) 2015 International Formulae Group. All rights reserved.

Mots clés : Culture, enquête, pratique culturale, cultivar, génétique.

\section{INTRODUCTION}

Le gingembre (Zingiber officinale Rosc.) est l'une des plus importantes épices à travers le monde. Cette espèce est d'une importance économique majeure à travers le monde (Nayak et al., 2005) ; car, elle permet de générer des ressources pour les producteurs. C'est une herbacée annuelle vivace grâce à son rhizome charnu, allongé et formé de plusieurs ramifications tubéreuses et noueuses. Selon Preeti et al. (2008), le gingembre est originaire du Sud-Est asiatique. C'est une large famille constituée de 47 genres et 1400 espèces (Parthasarathy et al., 2012). Kress et al. (2002) ainsi que Jatoi et al. (2007) rapportent que cette famille est divisée en 53 genres et plus de 1200 espèces qui se repartissent à travers l'Asie, les Iles du Pacifique et l'Afrique. Le gingembre est une épice d'une grande importance compte tenu de ses vertus médicinales. Cette espèce contient de nombreux composés tels que les 
gingéroles, les gingerdioles et les gingerdiones (Colleen et al., 2012). Ces composés possèdent une activité antioxydante élevée (Singh et al., 2008; Akbarian et al., 2011). Dans la médecine chinoise, le gingembre est traditionnellement utilisé pour traiter les problèmes d'estomac et d'indigestion, de diarrhée, de nausée (Shamsi et al., 2010; Azam et al., 2014), le rhumatisme, les maladies nerveuses, les maux de dents, l'asthme, la constipation, le diabète, etc... (Soong-Yukuo et al., 2011). La plante représente donc une plante d'intérêt majeur pour la pharmacopée.

Le gingembre a été introduit au Burkina Faso il y a plusieurs dizaines d'années et est communément appelé « gnamankou ». Il est cultivé essentiellement dans la partie Sud-Ouest du pays. La culture $\mathrm{du}$ gingembre est peu répandue et sa production est limitée sur de petites superficies malgré ses nombreuses vertus. Le gingembre est considéré comme une culture de rente. Il joue un rôle économique important pour les producteurs. Au Burkina Faso, les institutions en charge de l'agriculture ne disposent pas de données concernant la culture du gingembre et de plus le système de production ne respecte aucun itinéraire technique.

Cette étude vise à caractériser le système de production de la culture dans le but de mieux faire connaître l'espèce et de mettre en exergue ses potentialités.

\section{MATERIEL ET METHODES}

Identification des zones de production et investigation

La détermination de la zone de culture $\mathrm{du}$ gingembre s'est faite par une approche prospective réalisée avec l'appui des structures décentralisées du Ministère de l'Agriculture et de la Sécurité Alimentaire (MASA) du Burkina Faso. Ces structures sont les directions Provinciales ainsi que les Zones et Unités d'appui Techniques. A travers elles, nous avons identifié les localités productrices de gingembre. Une autre approche a consisté à se renseigner auprès des commerçants de gingembre dans des marchés locaux. Cela nous a permis d'obtenir des informations supplémentaires sur des villages de production de gingembre. Les différentes zones de culture retenues à l'issu de notre prospection ont été ensuite décrites sur la base des conditions climatiques, les groupes ethniques, les appellations locales des cultivars et leurs origines.

Des fiches d'enquête sous forme de questionnaires ont été élaborées et ont servi de guide d'entretien lors des enquêtes que nous avons réalisées auprès des producteurs de gingembre. Les enquêtes ont couvert au total vingt quatre (24) villages dans huit (08) départements dans les provinces du Kénédougou, de la Léraba et de la Comoé situées dans la partie Sud-Ouest du pays et appartenant à la zone Sud-soudanienne. Le questionnaire administré aux producteurs nous a permis de collecter des informations relatives aux pratiques culturales effectuées sur le gingembre. Ces informations ont été recueillies à l'aide de fiches descriptives pour chaque paysan enquêté. Les différents paramètres enregistrés ont été : le mode d'approvisionnement, l'origine et le nom local de la semence, la situation des champs, la préparation du sol, le mode de semis, les superficies emblavées, le cycle des cultivars, l'entretien des parcelles, la technique de conservation et les usages faits des rhizomes.

\section{Analyse des données}

Les données quantitatives enregistrées telles que la pluviométrie, le cycle des cultivars, et les superficies emblavées ont été regroupés en classes d'amplitudes égales. Les variables qualitatives (nom et origine des cultivars, mode de semis, entretien des parcelles, etc.) ont été traitées et classées. Les données ainsi préparées ont fait l'objet de calculs de fréquences et d'analyses statistiques descriptives des paramètres étudiés grâce au logiciel SPSS (Statistical Package for the Social Sciences) 17.0. 


\section{RESULTATS}

\section{Zones de production}

\section{Situation géographique}

Les enquêtes ont révélé que la culture du gingembre est pratiquée dans la partie SudOuest du Burkina Faso, essentiellement dans les régions des Cascades et des Hauts-Bassins. Dans ces régions, sa production se fait exclusivement dans les provinces de la Léraba, du Comoé et du Kénédougou (Figure 1). Ces trois (03) provinces font frontières avec le Mali et la Côte d'Ivoire respectivement à l'Ouest et au Sud. Elles sont limitées à l'Est par les provinces du Ioba, de la Bouguiriba et du Poni. Ces régions sont parmi les zones les mieux arrosées du pays. Les précipitations annuelles des cinq (05) dernières sont comprises entre 900 et 1300 $\mathrm{mm}$ avec des variations parfois considérables de la hauteur et de la distribution des pluies (Figure 2).

\section{Cycle des cultivars utilisés}

Les cultivars de gingembre utilisés ont des cycles très variables, allant de trois (03) à douze (12) mois (Figure 3). La majorité des accessions ont des durées de maturation de quatre (04) mois $(34,5 \%)$ et de cinq (05) mois (23\%).

\section{Appellations locales et significations}

Le gingembre est connu sous plusieurs appellations en fonction de la localité et de l'ethnie. A l'issu de nos enquêtes, il est ressorti que les appellations les plus courantes sont en langues sénoufo et dioula. Les appellations telles que dagban, dagbangué, dagbanagnoumougou et dougoumankanan dérivent de la sensation de chaleur ressentie sur la langue lors de la consommation du rhizome. Dagbangua qui désigne le gingembre "mâle" est dû à l'aspect très volumineux du rhizome avec un goût moins piquant. Dans le département de Niangoloko on rencontre des appellations telles que gnamangbassôlô, dougoumangbani, gnanbitan ou encore dagban qui signifient «piment de terre ». Le nom gnamankou en langue dioula est le nom générique de la plante et est également rencontré chez les sénoufos qui l'on emprunté.

\section{Mode d'obtention du matériel végétal}

Le matériel végétal utilisé par les producteurs provient de leurs villages de résidence ou des villages environnants. L'acquisition de la semence se fait soit par achat, sélection, don ou échange en nature. Le mode d'obtention le plus répandu est l'achat du rhizome avec 48,3\% des cas (Figure 4). Dans $43,7 \%$ des cas, les paysans sélectionnent les rhizomes à partir de leur propre production qu'ils conditionnent et utilisent comme semence à la campagne suivante. On rencontre également des cas de dons par des parents, amis ou connaissances et d'échanges en nature qui sont en très faibles proportions, respectivement $6,9 \%$ et $1,1 \%$ des cas.

\section{Pratiques culturales \\ Situation des champs et leurs superficies \\ Le gingembre est cultivé} essentiellement par les hommes. La culture se pratique dans les champs de brousse, généralement aux abords des bas-fonds et sur de petites superficies. On remarque que les producteurs dans la majeure partie des cas $(83,2 \%)$ produisent sur des superficies comprises entre 0,25 et 0,75 ha (Figure 5). Dans cet intervalle, $63,16 \%$ des producteurs adoptent des superficies plus petites $(0,25$ à 0,5 ha). En revanche, $11,4 \%$ des producteurs emblavent sur des superficies supérieures à un (01) ha.

Préparation du sol et mise en terre des semences

La préparation du sol se fait en fonction du type de terrain, qu'il soit sur une forte pente, plat ou engorgé d'eau. La confection de billon est le mode le plus répandu et cela se pratique dans $73,6 \%$ des cas. A l'aide d'une houe, les producteurs confectionnent des billons dont la largeur varie entre 01 et $02 \mathrm{~m}$ et la hauteur entre 30 et $50 \mathrm{~cm}$. Les billons sont espacés de 30 à $50 \mathrm{~cm}$ environ. Le labour à plat se pratique mais en faible proportion $(21,8 \%)$. On note également $4,6 \%$ des producteurs qui ont recours aux deux (02) pratiques (Figure 6). Le gingembre est une plante à multiplication végétative. $\mathrm{La}$ mise en terre se fait avec un morceau de 
rhizome utilisé comme semence. Les producteurs sélectionnent les meilleurs rhizomes qu'ils découpent en morceaux de 03 à $05 \mathrm{~cm}$ de longueur comportant 03 à 04 bourgeons viables. La sélection des rhizomes se fait sur la base de leur grosseur, l'aspect piquant que confère le rhizome sur la langue au goûter et de leur état sanitaire. La mise en terre des rhizomes s'effectue entre le mois d'avril et de juin, dans des poquets de 05 à 08 $\mathrm{cm}$ de profondeur espacés de 20 à $30 \mathrm{~cm}$ suivant le niveau de fertilisation du sol. Après la mise en terre, toute la parcelle est recouverte de paille constituée de résidus de récolte ou de branchage.

\section{Entretien des parcelles de culture}

Après les premières pluies, lorsque les tigelles apparaissent (03 semaines environ), le paillage est éliminé progressivement. Les parcelles de gingembre sont entretenues de façon continue pendant toute la période de développement. Les désherbages débutent à partir de la troisième semaine après la levée. Ils peuvent se faire par arrachage manuel ou par sarclage. Deux à trois sarclages sont nécessaires au cours du cycle de la culture selon le type d'adventices et le degré d'enherbement. Les producteurs ont très souvent recours aux fertilisants de nature diverses pour permettre une bonne croissance et un meilleur développement des plants. Les fertilisants utilisés sont de types organique et minéral (Figure 7). L'utilisation de la fumure organique et du NPK concerne $35,6 \%$ des cas. On a également une importante proportion $(26,4 \%)$ d'utilisation de fumure organique sans autre apport minéral. Les engrais organiques utilisés sont la bouse de vache, la fiente de poulet, les déjections des petits ruminants ou encore le compost issu des résidus de récoltes. Quant aux engrais minéraux, il s'agit essentiellement de l'urée et du NPK (Figure 7). Dans 18,4\% des cas, les producteurs utilisent la fumure organique, NPK et l'urée et $4,6 \%$ utilisent le NPK et l'urée. Certains producteurs $(4,6 \%)$ cultivent sans aucun apport d'engrais organique ni minéral. Les fertilisants organiques sont utilisés comme engrais de fond au labour afin d'enrichir le sol. Quant aux fertilisants minéraux, leur application se fait à des périodes diverses pendant la phase végétative de la plante. Ces engrais minéraux sont épandus à la volée.

\section{Récolte et conservation}

La maturation du rhizome est atteinte lorsque les feuilles et tiges se dessèchent. La récolte se fait à différentes périodes en fonction des besoins d'utilisation. Le nombre de mois qui s'écoulent entre la levée et la récolte des rhizomes varie entre trois (03) et douze (12) mois. Les périodes privilégiées se situent entre 4 et 7 mois. Après la récolte, la conservation des rhizomes se fait principalement de deux manières. Dans 83,9\% des cas (Figure 8), la conservation se fait dans des fosses où les paysans mettent les rhizomes puis ils recouvrent avec de la paille ou des feuilles séchées qu'ils arrosent au besoin. Cela se fait dans des endroits ombrageux. Dans le second cas $(14,9 \%)$, ils laissent le gingembre en terre dans leur champ qu'ils recouvrent d'un dense paillage afin de protéger les rhizomes contre l'effet du soleil.

\section{Usages et destination des récoltes}

Les paysans utilisent les rhizomes séchés en poudre comme épice dans la cuisine pour assaisonner les sauces et aussi dans le thé et le café. Le gingembre frais est épulché et broyé pour extraire le jus qui entre dans la fabrication de boissons rafraîssissantes. Les rhizomes sont également découpés en de fines tranches puis séchés et vendus sur la place du marché. Le gingembre a des vertues thérapeutiques. Il entre dans le traitement de certaines maladies telles que la toux, le rhume, les constipations, les hémorroïdes, etc. et est aussi utilisé comme aphrodisiaque. Le gingembre est considéré comme une culture de rente par les paysans. La plante est cultivée prioritairement pour la commercialisation. Les revenus sont utilisés pour satisfaire certains besoins notamment dans l'éducation des enfants et lors des cérémonies. 


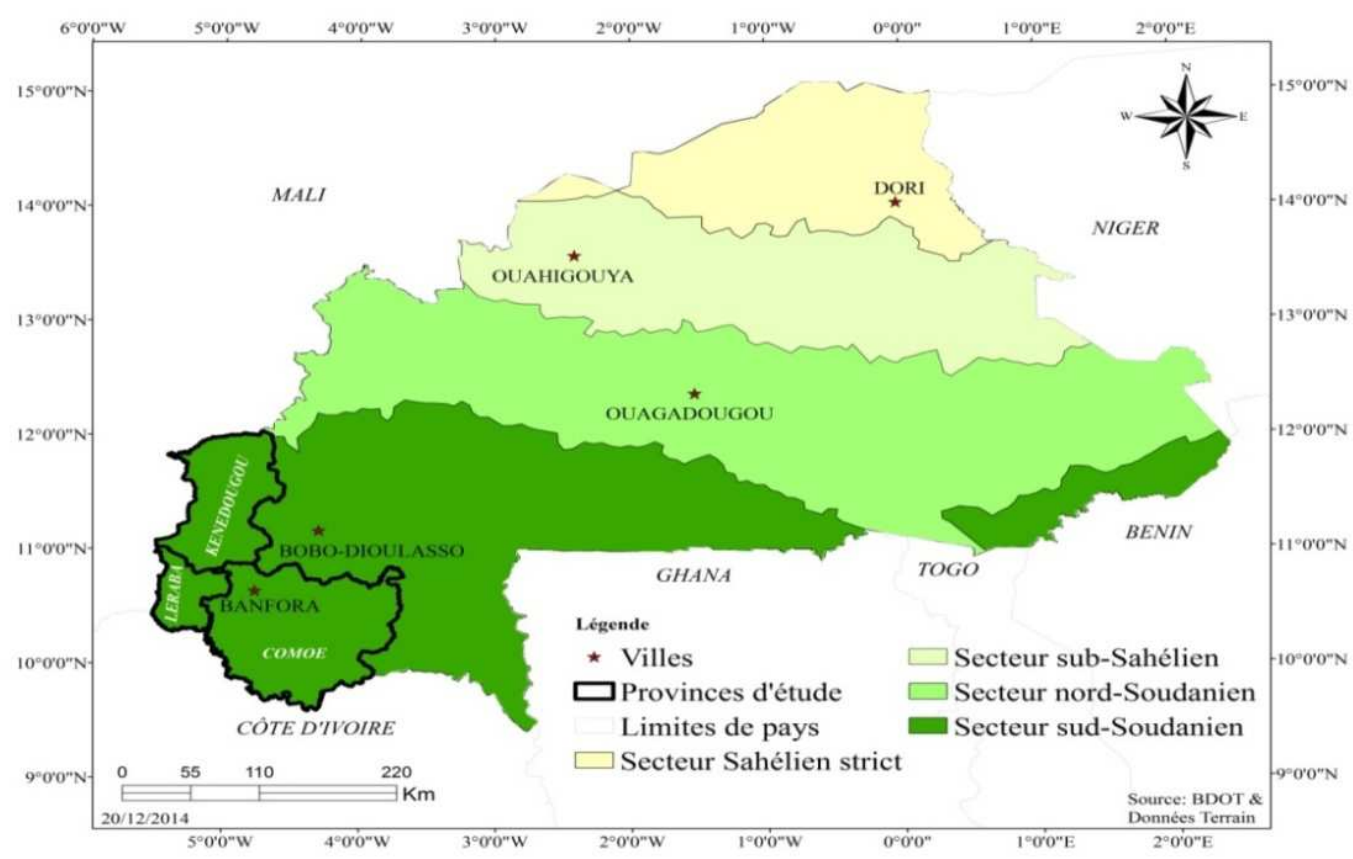

Figure 1 : Zone d'étude.

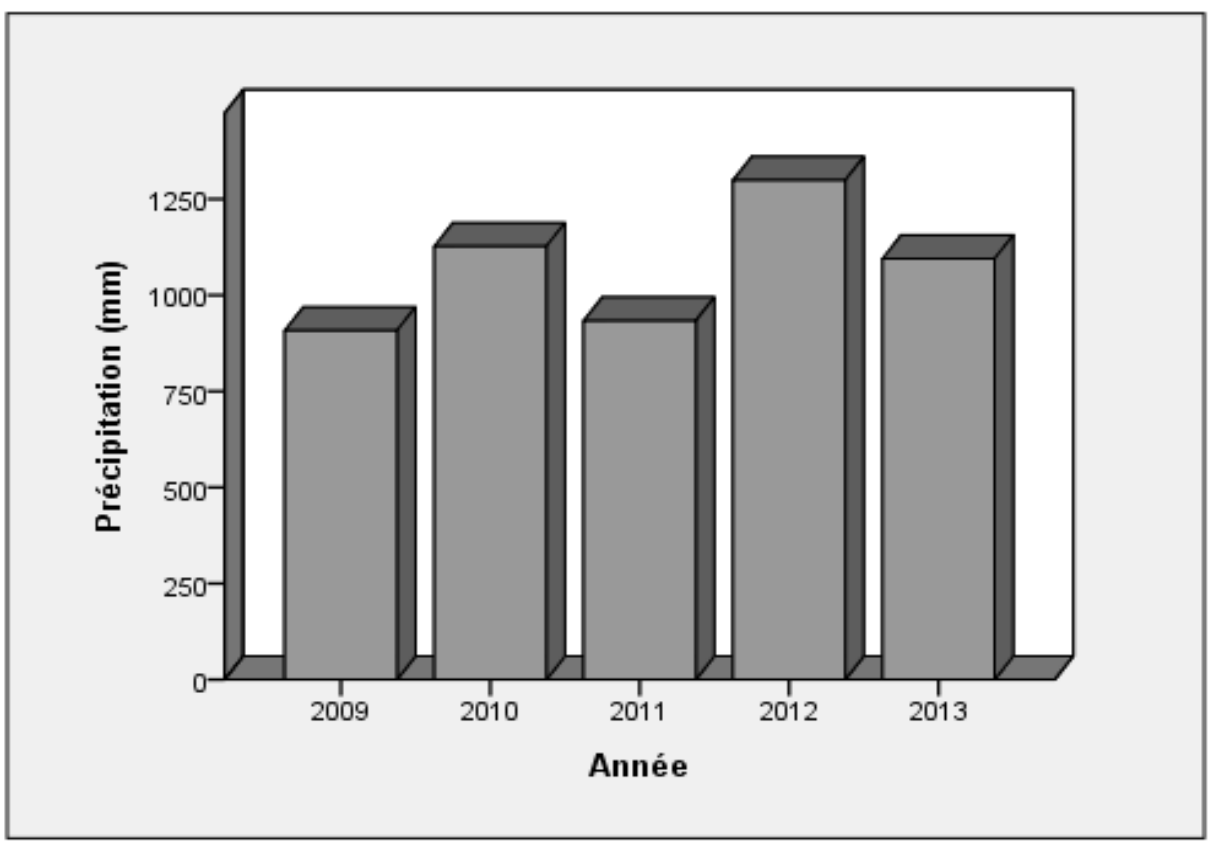

Figure 2: Pluviométrie de cinq (05) années écoulées dans la zone Sud-Ouest. Source : Direction Générale de la Météorologie du Burkina Faso (2014). 


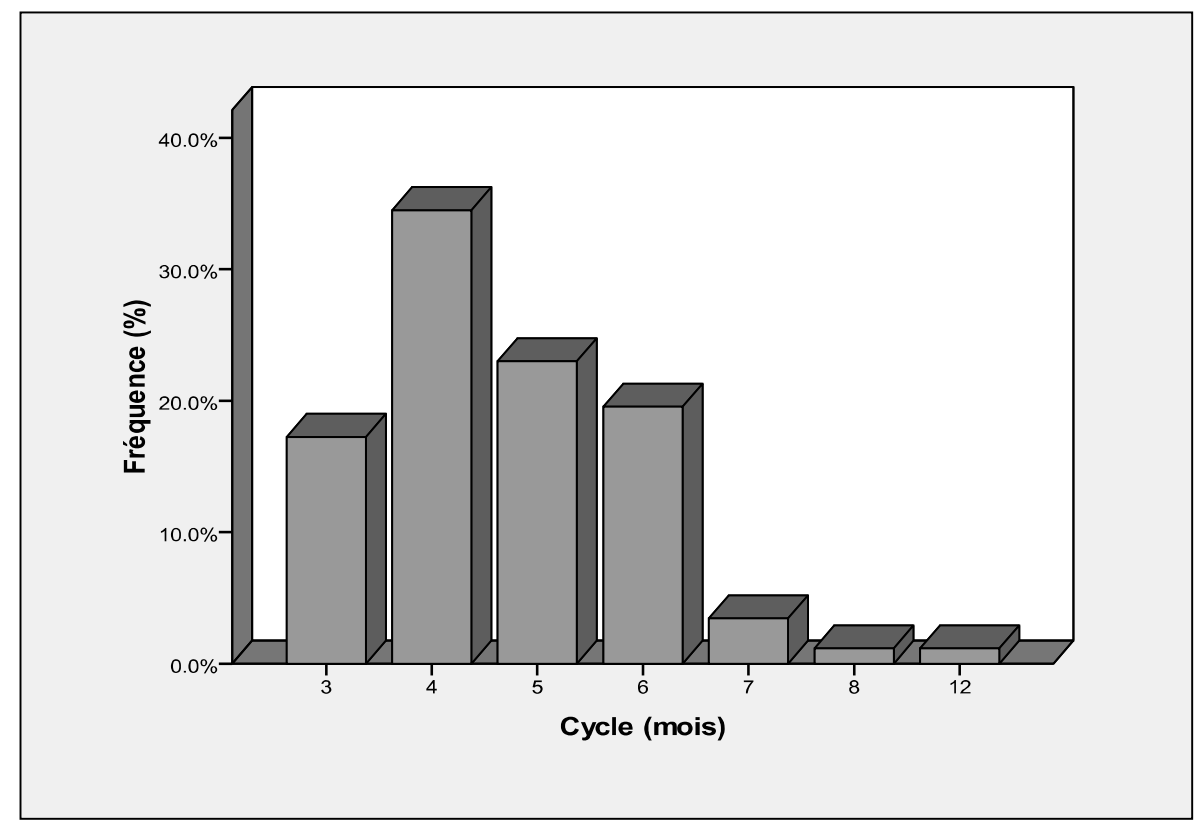

Figure 3: Cycles de maturation des cultivars.

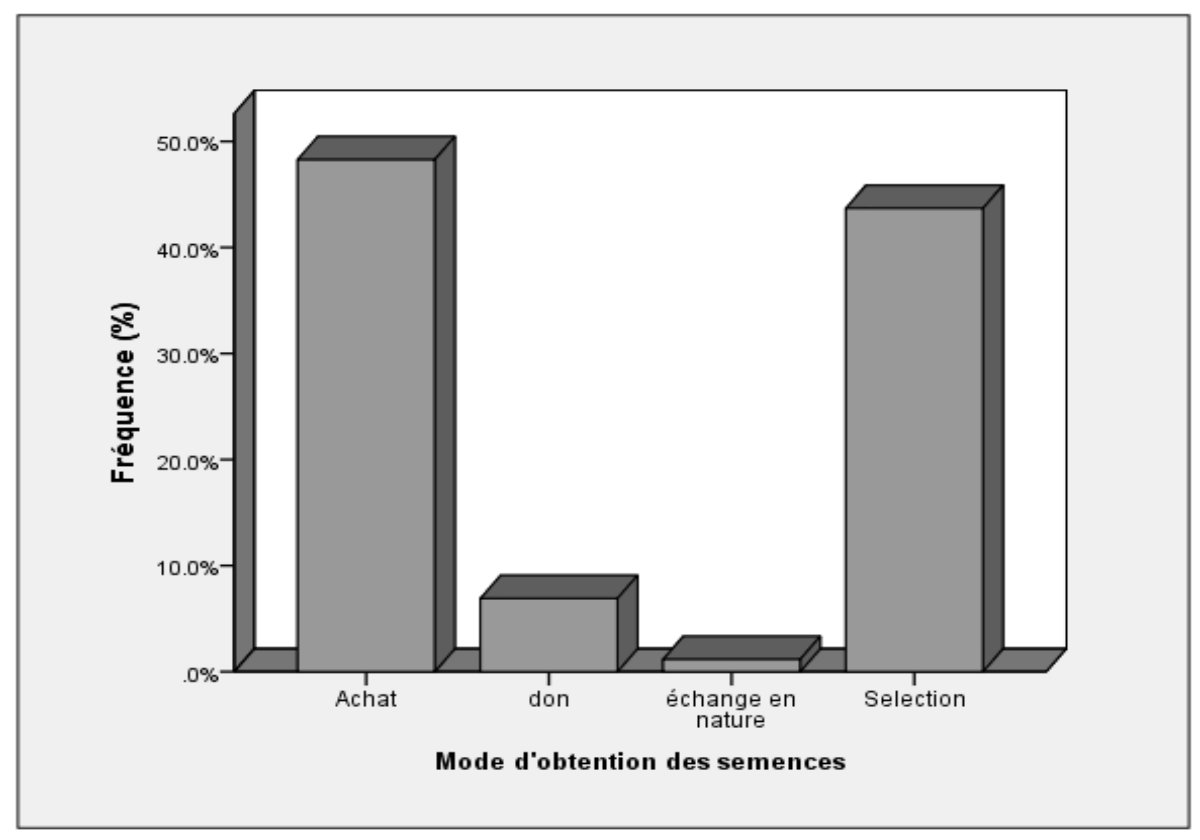

Figure 4: Mode d'obtention du matériel végétal. 


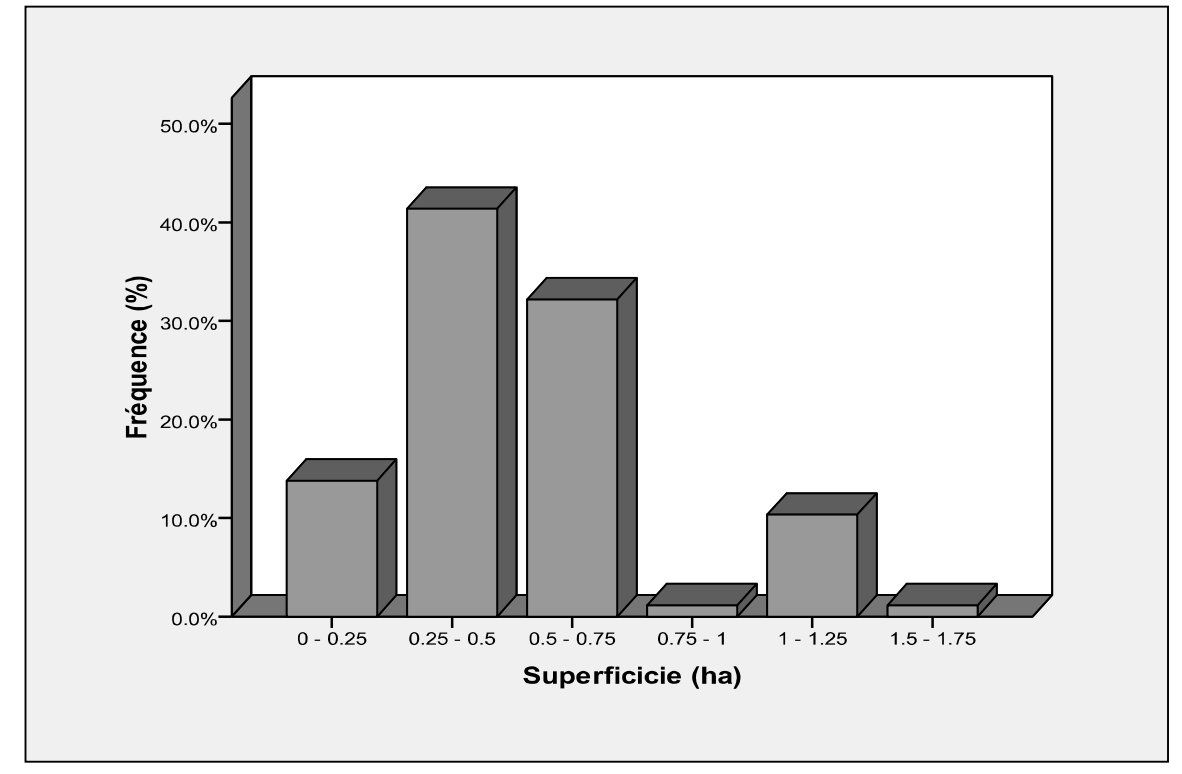

Figure 5: Superficies emblavées.

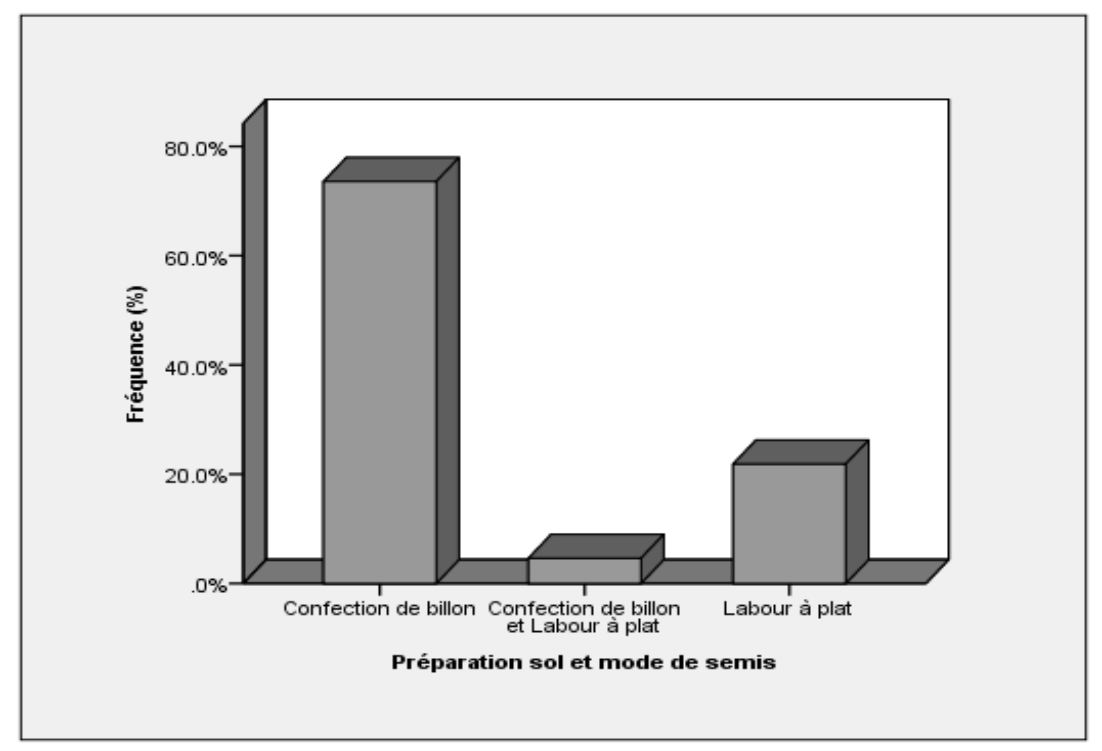

Figure 6: Préparation du sol et mode de semis. 


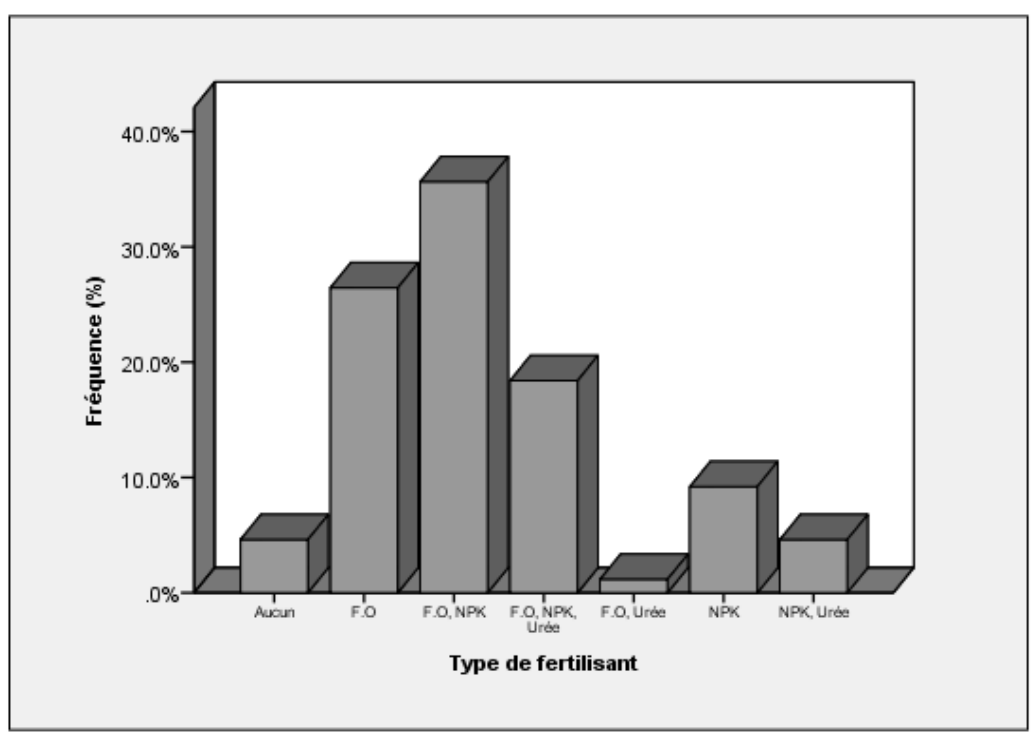

Figure 7: Types de fertilisants utilisés. F.O: fumure organique ; NPK (N: azote ; P: phosphate ; K:potassium).

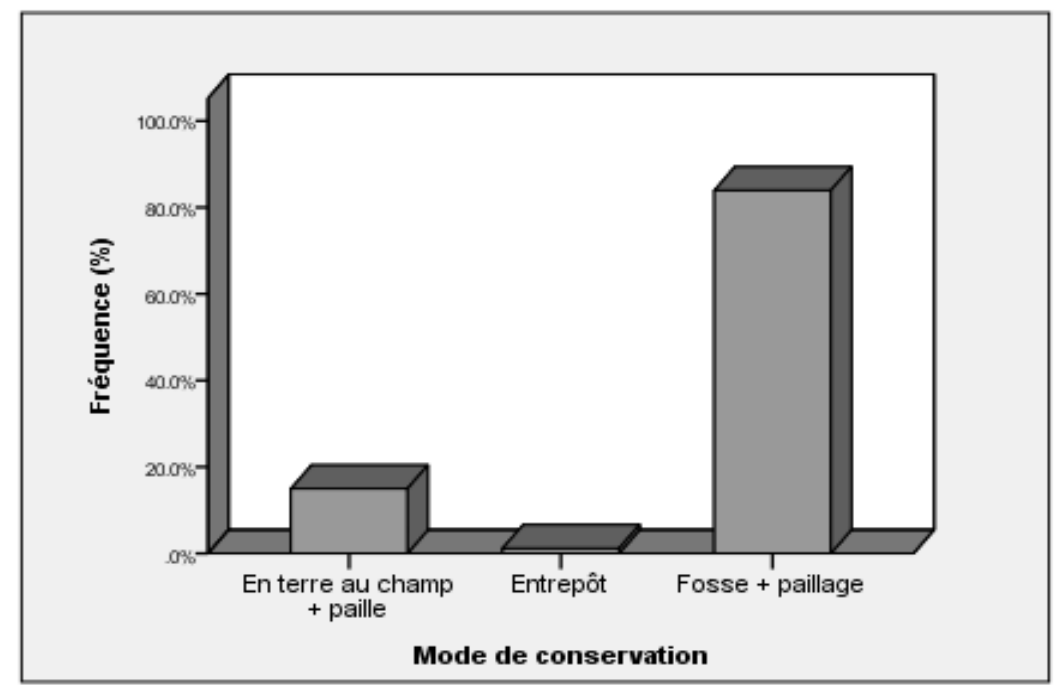

Figure 8: Modes de conservation des rhizomes.

\section{DISCUSSION}

Zone de production et conditions de culture La culture du gingembre se fait principalement dans les zones tropicales (Zheng et al., 2008 ; Ghosh et al., 2011) où la plante s'adapte le mieux. Le gingembre est maintenant cultivé dans presque tous les pays tropicaux et subtropicaux (Ravindra et al.,
2005 ; Xizhen et al., 2005) où les précipitations annuelles sont supérieures à 1000 mm (Nybe et Raj, 2005). Ces conditions présentent quelques similarités avec celles rencontrées dans la partie Sud-Ouest du Burkina Faso où on enregistre parfois près de 1300 mm d'eau par an. Au Burkina Faso, on ne dispose pas de données exactes à ce jour 
quant à la production de gingembre et les superficies emblavées. Le Burkina Faso reste un petit producteur de gingembre où les superficies des champs n'atteignent guère 2 ha. Le Nigéria est le premier pays producteur de gingembre en Afrique avec une production totale de $90000 \mathrm{t}$ sur une superficie de 174 000 ha (Peter et al., 2005). Malgré les vastes superficies emblavées, la production reste faible et cela est dû aux anciennes pratiques agronomiques, au manque d'utilisation de variétés améliorées, etc. (Ayodele et Sambo, 2014). Certains écotypes cultivés au Burkina Faso ont été introduits à partir des pays voisins notamment le Mali, la Côte d'Ivoire et le Ghana. Les cultivars dont les cycles sont supérieurs à 7 mois seraient introduits du Ghana et de la Côte d'Ivoire, où les pluies sont abondantes. Les écotypes qui ont des cycles compris entre 04 et 06 mois sont les plus utilisés car ils parviennent à boucler leur cycle. Selon Okwuowulu (2005), le rendement est étroitement lié à la durée des pluies qui ne doit pas excéder 06 mois. Des précipitations comprises entre 1500 et $3000 \mathrm{~mm} / \mathrm{an}$ avec une distribution de 8 à 10 mois seraient idéales pour la culture du gingembre (Rafie et Olczyk, 2003).

$\mathrm{Au}$ Burkina Faso, le gingembre est connu sous plusieurs appellations. Le nom gnamankou en langue locale Dioula est le nom le plus répandu sur l'ensemble du territoire national. Cette appellation est également utilisée en Côte d'Ivoire et au Mali. Les appellations en langue Sénoufo sont en rapport avec la taille du rhizome ou encore la sensation de chaleur que confère le rhizome sur la langue. Les cultivars les plus appréciés dans ces zones sont ceux dont le rhizome possède des doigts courts car ils ont un goût très piquant. La taille des rhizomes, la couleur de la peau, la taille des doigts du rhizome ainsi que les types de stries sur la peau du rhizome sont des traits morphologiques distinctifs entre les cultivars utilisés. Les différences observées entre les écotypes sont dues aux conditions agro-écologiques dans lesquelles ils évoluent (Joshi et al., 2012). Bien que le mode de multiplication du gingembre soit du type végétatif, il existe une grande variabilité au sein de l'espèce (Nayak et al., 2005). Kandiannan et al. (2009) rapportent que dans différents centres de production, il existe des cultivars distincts en raison du type de sol, le climat et les pratiques culturales. Le mode d'obtention le plus courant est l'achat des rhizomes. Les producteurs s'en procurent sur les marchés locaux. On retrouverait la même ressource génétique dans les mêmes villages.

\section{Pratiques culturales}

La culture du gingembre se pratique en culture pure au Burkina Faso. Parthasarathy (2012) rapporte que le gingembre peut être cultivé en association ou en culture pure. La multiplication du gingembre se fait par éclats de rhizome utilisés comme semence. La taille des semenceaux varient entre trois (03) et cinq (05) $\mathrm{cm}$ de long portant des bourgeons viables. Selon Parthasarathy (2012), les dimensions des rhizomes semences varient selon la variété et le type de terrain. Kandiannan et al. (1996) rapportent que la taille et le poids des rhizomes utilisés comme semence varient d'une localité à une autre et aussi en fonction de la variété utilisée. Pour la mise en place des parcelles les paysans confectionnent des billons. Aux abords des bas-fonds, la hauteur des billons est variable et atteignent parfois $50 \mathrm{~cm}$ ou plus afin d'éviter la pourriture des rhizomes ensemencés à cause de l'engorgement d'eau. Afin d'optimiser la production les producteurs mettent leur culture préférentiellement dans des endroits ombragés. Cela a été rapporté par Zhao et al. (2012) ; ils précisent que les plants de gingembre subissent une photo-inhibition lorsqu'ils sont soumis à un fort éclairage et se développent mieux lorsque l'éclairage est moins intense.

La culture du gingembre se pratique exclusivement en saison pluvieuse où les conditions climatiques sont propices au bon développement de la plante. Certains champs situés en bordure des points d'eau bénéficient d'un apport d'eau pour mieux boucler leur cycle lorsque les pluies s'écourtent souvent de 
façon inopinée. Les semis se font en quinconce avec une densité de $20-30$ x $20-$ $30 \mathrm{~cm}$. Ces densités diffèrent des pratiques en Inde dans la localité de Sikkim (30 x 45 - 60 $\mathrm{cm})$, en Australie (15 x $40-60 \mathrm{~cm})$, en Chine (20 x 60-65 cm) et en Jamaïque $(15-20 \times 15$ $-20 \mathrm{~cm}$ ) (Parthasarathy, 2012). Les semis se font généralement dès le démarrage des pluies en mai et début juin. Certains producteurs mettent leurs semenceaux en terre à partir du mois d'avril. Xizhen et al. (2005) rapportent qu'en Chine les semis se font entre les mois de janvier et avril. Lorsque les rhizomes sont mis en terre précocement, l'émergence des plantules a lieu dès le démarrage des pluies. Cela favorise l'enracinement des plants de façon précoce et leur permet de résister aux pluies diluviennes (Randhawa et Nandpuri , 1970).

Après le semis, le paillage pratiqué traditionnellement permet de protéger les rhizomes en terre contre l'effet de l'ensoleillement, de garder l'humidité du sol, améliore la fertilité du sol en apportant de la matière organique et réduit également la prolifération des mauvaises herbes. Ces avantages ont été signalés par Parthasarathy (2012) qui souligne également que le paillage améliore la germination et l'infiltration de l'eau de ruissèlement. Les mauvaises herbes constituent un problème majeur dans la production du gingembre car elles réduisent considérablement les rendements. L'entretien des parcelles consiste essentiellement à faire des désherbages manuels et à appliquer de l'engrais pour permettre un bon développement des plants. Les engrais minéraux ne sont pas à la portée de tous les producteurs du fait de leurs coûts élevés. Lorsque ces engrais sont utilisés, leur application ne suit pas un itinéraire technique établi, car les périodes d'application et les doses sont très variables d'un producteur à un autre. Selon Parthasarathy (2012), les besoins en fertilisants varient selon les cultivars, le type de sol et les conditions climatiques. Les doses $(\mathrm{Kg} / \mathrm{ha})$ d'éléments minéraux utilisés dans les différents Etats de l'Inde sont 36 $225 \mathrm{~N}: 8-50 \mathrm{P}$ : 40-166 K. Au Nigeria et dans la partie occidentale indienne, les doses recommandées sont respectivement 105 : 60 : 105 (Okwuowulu, 2005).

En fonction des besoins d'utilisation, la récolte se fait sur une période allant de 3 à 7 mois après la levée. Au Nigéria, la maturité a lieu après 7 à 8 mois selon qu'il s'agisse du gingembre noir ou jaune respectivement (Okwuowulu, 2005). Lorsque le gingembre est utilisé comme légume, les rhizomes sont récoltés à partir du sixième mois tandis que pour la préparation de gingembre $\mathrm{sec}$, la récolte a lieu après huit mois quand les feuilles jaunissent et commence à sécher, rapportent Jayashree et al. (2012). Le meilleur moment de récolte se situent au septième mois selon Ramana et al. (2003); car à cette période, les rhizomes présentent une forte quantité d'oléorésine. Afin de préserver le matériel végétal et d'assurer la conservation de son essence génétique, les producteurs ont recours à une méthode très simple pour la conservation des rhizomes sur de longues périodes. Ils n'utilisent aucun produit chimique bien que les rhizomes soient susceptibles d'attaques parasitaires et de maladies. Hiremath (2006) affirme que les rhizomes sont très souvent attaqués par les agents pathogènes tels que Pseudomonas solanacearum et Phythium sp. En fonction de la quantité de gingembre, les producteurs creusent une fosse à l'ombre dans lequel ils placent les rhizomes. Lorsque les rhizomes sont conservés à des températures comprises entre 22 et $25{ }^{\circ} \mathrm{C}$, ils gardent leur pouvoir germinatif et ont une forte vigueur (Parthasarathy, 2012). La conservation des rhizomes au champ consiste à laisser les rhizomes en terre et à faire un paillage conséquent afin de les protéger contre l'effet des rayons du soleil.

\section{Destination des récoltes et leurs usages}

Le gingembre produit au Burkina Faso ne fait pas l'objet de commerce international. Sa production reste limitée et en revanche il est importé à partir des pays voisins. Le rhizome est utilisé moulu ou frais pour assaisonner les sauces. Le jus extrait du 
rhizome entre dans la fabrication de boissons rafraîchissantes lors des cérémonies. Le rhizome séché et moulu est très apprécié dans le café et le thé. Okonta et al. (2008) affirment qu'il est utilisé comme épice ou condiment et représente aussi un agent phyto-thérapeutique. Le gingembre est utilisé pour la fabrication de boissons telles que la bière, la liqueur et dans la conservation de certains produits (Hiremath, 2006). Dans les marchés locaux on rencontre le gingembre transformé. Les rhizomes découpés en de fines tranches, séchés et saupoudrés de sucre sont consommés sous forme d'amuse-gueule. Le gingembre est utilisé pour le traitement de certains maux tels que le rhume, les constipations, les nausées, les hémorroïdes, les angines, les maux de ventre, la toux, etc. Il est également utilisé dans la pharmacopée chinoise selon Vendruscolo et al. (2006) pour le traitement de nombreuses maladies. $\mathrm{Au}$ Nigéria, Okonta et al. (2008) rapportent que le gingembre est utilisé par la majeure partie des populations dans des mets variés et contre les maux gastro-intestinaux. Zheng et al. (2008) rapportent que le gingembre était cultivé principalement dans les zones tropicales asiatiques depuis les temps anciens pour soulager le rhume, les fièvres, les problèmes de digestion et stimule l'appétit.

\section{Conclusion}

La culture du gingembre se pratique essentiellement dans la partie Sud-Ouest du Burkina Faso avec des précipitations annuelles comprises entre 900 et $1200 \mathrm{~mm} / \mathrm{an}$. $\mathrm{Sa}$ production exige des conditions pédoclimatiques assez particulières. La culture de la plante reste limitée ; elle se pratique sur de petites superficies aux alentours des basfonds avec des moyens dérisoires entraînant une faible production. La productivité du gingembre est liée à des facteurs tels que la qualité du rhizome semence ainsi que les conditions biotiques et abiotiques. L'étude du système de culture du gingembre montre des limites de sa production. Cette restriction est essentiellement due à un manque d'itinéraire technique établit pour la production du gingembre, aux exigences de la plante sur le plan climatique et à la pénibilité du travail par rapport à sa rentabilité. Une meilleure connaissance des différents systèmes de culture du gingembre combiné judicieusement aux approches technologiques nouvelles permettrait d'améliorer le système de production et d'accroitre la productivité de la plante. Des investigations plus poussées se structurant autour de la préservation des ressources génétiques sont nécessaires afin de mieux connaître les différents cultivars rencontrés sur l'ensemble du territoire.

\section{REMERCIEMENTS}

Nos remerciements vont à l'endroit du Fond National de la Recherche et de l'Innovation pour le Développement (FONRID) pour son support financier.

\section{REFERENCES}

Abdollah A, Abolghasem G, Ardashir SA, Hossein M. 2011. Effects of ginger root (Zingiber officinale) on egg yolk cholesterol, antioxidant status and performance of layinghens. J. Appl. Ani. Res., 39: 10-21.

Ayodele TJ, Sambo BE. 2014. Ginger (Zingiber officinale Roscoe) production efficiency and constraints among small scale farmers in southern Kaduna, Nigeria. J. Agric. Sci., 6(8): 141-148.

Azam R, Jabeen A, Alam T, Mushtaq S, Mohmad SH. 2014. Zanjabil (Zingiber officinalis): A Review. J. Pharm. Sci. Innov., 3(4): 278-282.

Colleen NAS, Bailey-Shaw YA, Hibbert SL, Green C, Smith AM, Williams LAD. 2012. Characterization of cultivars of Jamaican ginger Zingiber officinale Roscoe) by HPTLC and HPLC. Food Chem., 131(4): 1517-1522.

Hiremath RC. 2006. Micropropagation of ginger (Zingiber officinale Rosc.). Master thesis, University of Agricultural Sciences, Dharwad, p. 40.

Jatoi SA, Kikuchi A, Mimura M, Yi SS, Watanabe N. 2008. Relationship of Zingiber species and genetic variability 
assessment in ginger ( $Z$. officinale) accessions from ex-situ genebank, onfarm and rural markets. Breed. Sci., 58: 261-270.

Jayashree E, Visvanathan R, John ZT. 2012. Quality of dry ginger (Zingiber officinale) by different drying methods. J. Food Sci. Tech., DOI 10.1007/s13197012-0823-8.

Joshi RK, Mohanty S, Kar B, Nayak S. 2012. Assessment of Genetic Diversity in Zingiberaceae Through Nucleotide Binding Site-Based Motif-Directed Profiling. Biochem. Genet., 50: 642-656.

Kandiannan K, Parthasarathy U, Krishnamurthy KS, Thankamani CK, Srinivasan V. 2009. Modeling individual leaf area of ginger (Zingiber officinale Roscoe) using leaf length and width. Sci. Hort., 120: 532-537.

Kress WJ, Prince LM, Williams KJ. 2002. The phylogeny and a new classification of the gingers (Zingiberaceae): evidence from molecular data. Americ. J. Bot., 89: 1682-1696.

Nayak S, Naik PK, Qcharya L, Mukherjee AK, Panda PC, Das P. 2005. Assessment of genetic diversity among 16 promising cultivars of ginger using cytological and molecular markers. Z. Naturforsch, 60c: 485-492.

Nybe EV, Raj NM. 2005. Ginger production in India and other South Asian countries. In Medicinal and Aromatic PlantsIndustrial profiles: Ginger, the Genus Zingiber, Ravindran PN, Babu KN (eds). CRC Press: Washington DC; 211-240.

Okonta JM, Uboh M, Obonga WO. 2008. Herb-drug interaction: A case study of effect of ginger on the pharmacokinetic of metronidazole in rabbit. Indian. $J$. Pharm. Sci., 70: 230-242.

Okwuowulu PA. 2005. Ginger in Africa and the Pacific Ocean Island. In Medicinal and Aromatic Plants-Industrial Profiles: Ginger, the Genus Zingiber, Ravindran PN, Babu KN (eds). CRC Press: Washington DC; 279-304.
Parthasarathy VA, Srinivasan V, Nair RR, John ZT, Kumar A, Prasath D. 2012. Ginger: Botany and Horticulture. Indian Institute of Spices Research Indian Council of Agricultural Research: Kerala, India.

Peter KV, Nybe EV, Kurien A. 2005. Yield gaps and constraints in ginger. In Medicinal and Aromatic PlantsIndustrial Profiles: Ginger, the Genus Zingiber, Ravindran PN, Babu KN (eds). CRC Press: Washington DC; 527-532.

Preeti C, Dnyaneshwar W, Kalpana J, Bhushan P. 2008. Development of SCAR (sequence-characterized amplified region) markers as a complementary tool for identification of ginger (Zingiber officinale Roscoe) from crude drugs and multi component formulations. Biotech. Appl. Biochem., 50: 61-69.

Rafie AR, Olczyk T. 2003. Hydroponic production of fresh ginger roots as an alternative method for south Florida. Proc. Fla. State Hort. Soc., 116: 51-52.

Ramana KV, Shiva KN, Johny AK. 2003. Production of quality planting materials of ginger. National consultative meeting for improvement in productivity and utilization of ginger, Aizawl, Mizoram, India, 37-45.

Ravindran PN, Babu NK, Shiva KN. 2005. Botany and crop improvement In Medicinal and Aromatic PlantsIndustrial Profiles: Ginger, the Genus Zingiber, Ravindran PN, Babu KN (eds). CRC Press: Washington DC; 15-86.

Shariq S, Tajuddin, Afaq SH. 2010. Spice and Medicine: Zingiber Officinale. Int. J. Appl. Biol. Pharm. Tech., 1(3): 968-973.

Singh G, Kapoor IPS, Singh P, Heluani CS, Lampasona MP, Catalan CAN. 2008. Chemistry, antioxidant and antimicrobial investigations on essential oil and oleoresins of Zingiber officinale. Food Chem. Toxicol., 46: 3295-3302.

Soong Y, Rong JL, Yu TY, Jun YL, Chung YC. 2011. A new phenylalkanoid from 
the rhizomes of Zingiber officinale. Nat. Prod. Res., 26: 1318-1322.

Vendruscolo A, Takaki I, Bersani-Amado LE, Dantas JA, Bersani-Amado CA, Cuman RKN. 2006. Anti-inflammatory and antinociceptive activities of Zingiber officinale Roscoe essential oil in experimental animal models. Indian $J$. Pharm., 38: 58-59.

Xizhen A, Jinfeng S, Xia X. 2005. Ginger production in Southeast Asia. In Medicinal and Aromatic PlantsIndustrial Profiles: Ginger, the Genus
Zingiber, Ravindran PN, Babu KN (eds). CRC Press: Washington DC; 241-278.

Zhao W, Wang S, Huang XLH, Sui X, Zhan Z. 2012. Molecular cloning and characterization of the light-regulation and circadian-rhythm of the VDE gene promoter from Zingiber officinale. Plant Cell Rep., 31: 1381-1392.

Zheng Y, Liu Y, Ma M, Xu K. 2008. Increasing in vitro microrhizome production of ginger (Zingiber officinale Roscoe). Acta Physiol. Plant, 30: 513519. 